\title{
Coil spring fiducial markers placed safely using navigation bronchoscopy in inoperable patients allows accurate delivery of CyberKnife stereotactic radiosurgery
}

\author{
Carsten Schroeder, MD, PhD, ${ }^{\mathrm{a}}$ Rana Hejal, MD, ${ }^{\mathrm{b}}$ and Philip A. Linden, MD ${ }^{\mathrm{a}}$
}

\begin{abstract}
Objectives: CyberKnife stereotactic body radiosurgery is a potentially curative option for medically inoperable Stage I lung cancer. Fiducial marker placement in or near the tumor is required. Transthoracic placement using computed tomography guidance has been associated with a high risk of iatrogenic pneumothorax. Electromagnetic navigation bronchoscopy offers a safer method of placing markers; however, previous studies using linear markers have shown at least a $10 \%$ dislocation rate. We describe the use of coil-spring fiducial markers placed under moderate sedation in an outpatient bronchoscopy suite.
\end{abstract}

Methods: A total of 52 consecutive nonoperative patients with isolated lung tumors underwent fiducial placement using electromagnetic navigation bronchoscopy. Of the 52 patients, 4 received 17 linear fiducial markers, and 49 patients with 56 tumors received 217 coil-spring fiducial markers. The procedures were considered successful if the fiducial markers had been placed in or near the tumors and had remained in place without migration, allowing radiosurgery without the need for additional fiducial markers.

Results: A total of 234 fiducial markers were successfully deployed in 52 patients with 60 tumors (mean diameter $23.7 \mathrm{~mm})$. Of these 60 tumors, $35(58 \%)$ were adjacent to the pleura. At CyberKnife planning, $8(47 \%)$ of 17 linear fiducial markers and $215(99 \%)$ of 217 coil-spring fiducial markers $(P=.0001)$ were still in place. Of the 4 patients with linear fiducial markers, 2 required additional fiducial placements; none of the patients with coil fiducial markers required additional procedures. Three pneumothoraces $(5.8 \%)$ occurred in peripheral lesions ( 2 were treated with a pig-tail chest tube and 1 with observation only).

Conclusions: Deployment of coil spring fiducial markers using navigation bronchoscopy can safely be performed with the patient under moderate sedation with almost no migration and a $5.8 \%$ rate of pneumothorax.

(J Thorac Cardiovasc Surg 2010;140:1137-42)

Patients with lung cancer often have severe smokinginduced morbidities such as chronic obstructive pulmonary disease (COPD) and arteriosclerotic heart disease that precludes a curative surgical approach. Stereotactic body radiosurgery is a treatment option for patients who are unable to undergo lung resection. ${ }^{1-5}$ For precise tumor ablation, the CyberKnife (CyberKnife, Accuray Inc, Sunnyvale, Calif) system requires fiducial marker placement in or near the target tumor. Fiducial placement under transthoracic computed tomography (CT) guidance is associated with a high risk of iatrogenic pneumothorax development of at least $13 \%{ }^{6}$ This rate is likely conservative because

\footnotetext{
From the Division of Thoracic and Esophageal Surgery, ${ }^{\mathrm{a}}$ and the Pulmonary/Critical Care and Sleep Medicine, ${ }^{\mathrm{b}}$ Case Medical Center University Hospitals, Cleveland, Ohio.

Disclosures: None.

Read at the 90th Annual Meeting of The American Association for Thoracic Surgery, Toronto, Ontario, Canada, May 1-5, 2010.

Received for publication April 27, 2010; revisions received July 19, 2010; accepted for publication July 30, 2010; available ahead of print Sept, 20, 2010.

Address for reprints: Carsten Schroeder, MD, PhD, Division of Thoracic and Esophageal Surgery, University Hospitals, Case Medical Center, 11100 Euclid Ave., Cleveland, OH 44106-5011 (E-mail: Carsten.Schroeder@UHhospitals.org). $0022-5223 / \$ 36.00$

Copyright (c) 2010 by The American Association for Thoracic Surgery doi:10.1016/j.jtcvs.2010.07.085
}

CT-guided lung biopsy specimens have revealed pneumothorax rates of $23 \%$ to $38 \%,,^{7,8}$ and a recent study reported a $48 \%$ pneumothorax rate with CT-guided fiducial marker placement. ${ }^{9}$ Electromagnetic navigation bronchoscopy (ENB) offers a less morbid alternative to accurately guide bronchoscopic tools and deploy fiducial markers within the bronchial tree to endoscopically invisible peripheral lung lesions. Previous studies, in which linear markers were used, showed at least a $10 \%$ dislocation rate ${ }^{10,11}$ and required general anesthesia for placement. $^{11,12}$ In the present study, we have proposed the use of coil-spring fiducial markers deployed using ENB with the patient under moderate sedation in an outpatient bronchoscopy suite.

\section{MATERIALS AND METHODS}

A total of 52 consecutive patients who had been referred for bronchoscopic fiducial marker placement were recruited between September 2008 and March 2010. All patients had peripheral lung tumors and no CT evidence of endobronchial pathologic features. All cases were reviewed at a multidisciplinary thoracic oncology board. Of the 52 patients, 47 had been deemed inoperable and 5 had refused surgery. All patients underwent positron emission tomography scanning, and if any activity was seen in the mediastinum or hilum, the patients underwent either mediastinoscopy or endobronchial ultrasonography to rule out extrapulmonary disease before proceeding with stereotactic body radiosurgery. The institutional review 


\section{Abbreviations and Acronyms \\ $\mathrm{COPD}=$ chronic obstructive pulmonary disease \\ $\mathrm{CT}=$ computed tomography \\ $\mathrm{ENB}=$ electromagnetic navigation \\ bronchoscopy \\ $\mathrm{SD}=$ standard deviation}

board of the Case Comprehensive Cancer Center approved the data collection and analysis.

\section{ENB Procedure}

The superDimension inReach System (superDimension Inc, Plymouth, Minn) was used for ENB. All patients underwent noncontrast-enhanced CT scans of the chest with a slice thickness of 1 to $1.5 \mathrm{~mm}$ and a slice interval of 1 to $2 \mathrm{~mm}$, with an overlap of $1 \mathrm{~mm}$. The initial planning phase required importing Digital Imaging and Communications in Medicine format CD data into the planning software. Using 3-dimensional reconstruction, an endobronchial track to the tumor target lesion was mapped. The patient was placed on an electromagnetic location board. Bronchoscopy was performed with the patient under moderate sedation using the oral route and an adult therapeutic bronchoscope (Olympus IT160; Olympus, Tokyo, Japan) with a 2.8 -mm working channel. The patient's endobronchial anatomic landmarks were registered in the magnetic field and overlayed with the 3-dimensional reconstructed CT scan. An anatomic divergence error of $<5 \mathrm{~mm}$ was accepted. Navigation began by wedging the bronchoscope in the suspected bronchial segment and steering the sensor probe and the extended working channel to the tumor using the multiplanar CT scan images and the "tip-view" orientation. The tumor was considered located when a minimum distance $(<1 \mathrm{~cm})$ between the steerable probe tip and the center of the lesion was achieved (Figure 1). When navigation was completed, the steerable probe was removed, and the extended working channel sheath left in place. A transbronchial biopsy could be performed at this point directly into the tumor.

\section{Fiducial Marker Placement}

The initial 4 patients received linear gold fiducial markers $(1 \times 5 \mathrm{~mm}$, CyberMark MT-NW-887-853, Civco Medical Solutions, Orange City, Iowa). The subsequent 56 fiducial marker placements used platinum coil spring markers (diamond-shaped, $3 \times 3.3 \mathrm{~mm}$, VortX-18, Boston Scientific, Cork, Ireland) (Figure 2). Both fiducial markers were delivered using wax tip microbiology specimen brushes (catalogue no. 130; ConMed Endoscopic Technologies, Chelmsford, Mass) (Figure 2, A) through the extended working channel sheath under fluoroscopic control. The wax plug was removed before loading the delivery brush with either fiducial type and than temporarily sealed with viscose sterile lubricant. The VortX-18 fiducial markers were loaded into the microbiology catheter by way of injection from their delivery needle. After each fiducial placement, the working channel sheath was navigated to a different area of the tumor before the next marker was deployed. Ideally, the fiducial markers should have a minimum spatial separation of $20 \mathrm{~mm}$ and a 3-dimensional angular separation. Chest radiographs were obtained after the procedure to confirm the fiducial position and to rule out any pneumothorax (Figure 3).

CyberKnife planning CT scans were performed 7 to 14 days after fiducial marker placement and reviewed by a thoracic surgeon, radiation oncologist, and nuclear physicist to assess the adequacy of fiducial marker placement. The need for alternative or additional intrathoracic fiducial placement was documented as procedure failure.

Data collection, demographics, and basic statistical analysis were performed on a personal computer using the Statistical Package for Social
Sciences for Windows, version 13.0 (SPSS Inc, Chicago, Ill). The parametric data are presented as the in mean \pm standard deviation (SD). An association between categorical variables was tested using the 2-tailed Fisher exact test.

\section{RESULTS}

A total of 52 patients underwent 60 fiducial maker placements. A total of 234 fiducial markers were successfully deployed. The initial 4 patients had linear gold fiducial markers placed. Of the 4 patients, 2 required additional CT or bronchoscopy-guided fiducial placement. Only 8 $(47 \%)$ of the 17 linear fiducial markers were still in place at CyberKnife planning 1 to 2 weeks after placement. This led us to change the type of fiducial markers to coil spring platinum markers that promised better anchorage within the lung tissue because of their self-expanding mechanism and thrombogenic filament attachments (Figure 2, $B)$. Of the 217 coil-spring fiducial markers, $215(99 \%)$ were still in place at CyberKnife planning, a significant improvement compared with the linear fiducial type $(P=.0001)$. All the coil spring fiducial marker patients could be treated with CyberKnife radiosurgery, and none needed any additional fiducial marker placement.

The study group included 32 women and 20 men, with a mean age of 73.8 years (range, 50-94 years). One patient underwent fiducial marker placement twice, first with linear and later successfully with coil fiducial markers. Seven patients had 2 tumor locations. Of the 60 lesions, 51 were known non-small cell lung cancer or were discovered at ENB by on-site biopsy assessment, 4 were proven metastases, and 5 were histologically unknown at ENB despite onsite biopsy assessment. The mean tumor diameter was $23.7 \pm 10.2 \mathrm{~mm}$ (range, $8-53 \mathrm{~mm}$ ). Of the 60 tumors, 35 $(58 \%)$ were adjacent to the pleura. A total of 21 patients $(35 \%)$ underwent concurrent transbronchial biopsy at ENB. The mean bronchoscopy procedure duration was $37 \pm 16.2$ minutes (range, 15-70 minutes), and the mean pulse-technique fluoroscopy time was $66 \pm 31$ seconds (range, 36-152 seconds).

Of the 52 patients, 3 developed pneumothorax (5.8\%), 2 of which were associated with transbronchial biopsy. Two of the patients were treated with pig-tail chest tube placement in the bronchoscopy suite and one was observed. A postprocedural survey was performed, and no additional complications (eg, hemorrhage, pneumonia, hospital admissions) were recorded. No procedure-related delays in CyberKnife treatment were encountered.

\section{DISCUSSION}

Many patients with early-stage lung cancer, because of significant comorbidities such as COPD, severe cardiacrelated disease, or other disease, cannot undergo surgical resection. External beam radiotherapy has been used for many of these medically inoperable patients, with cure rates of 


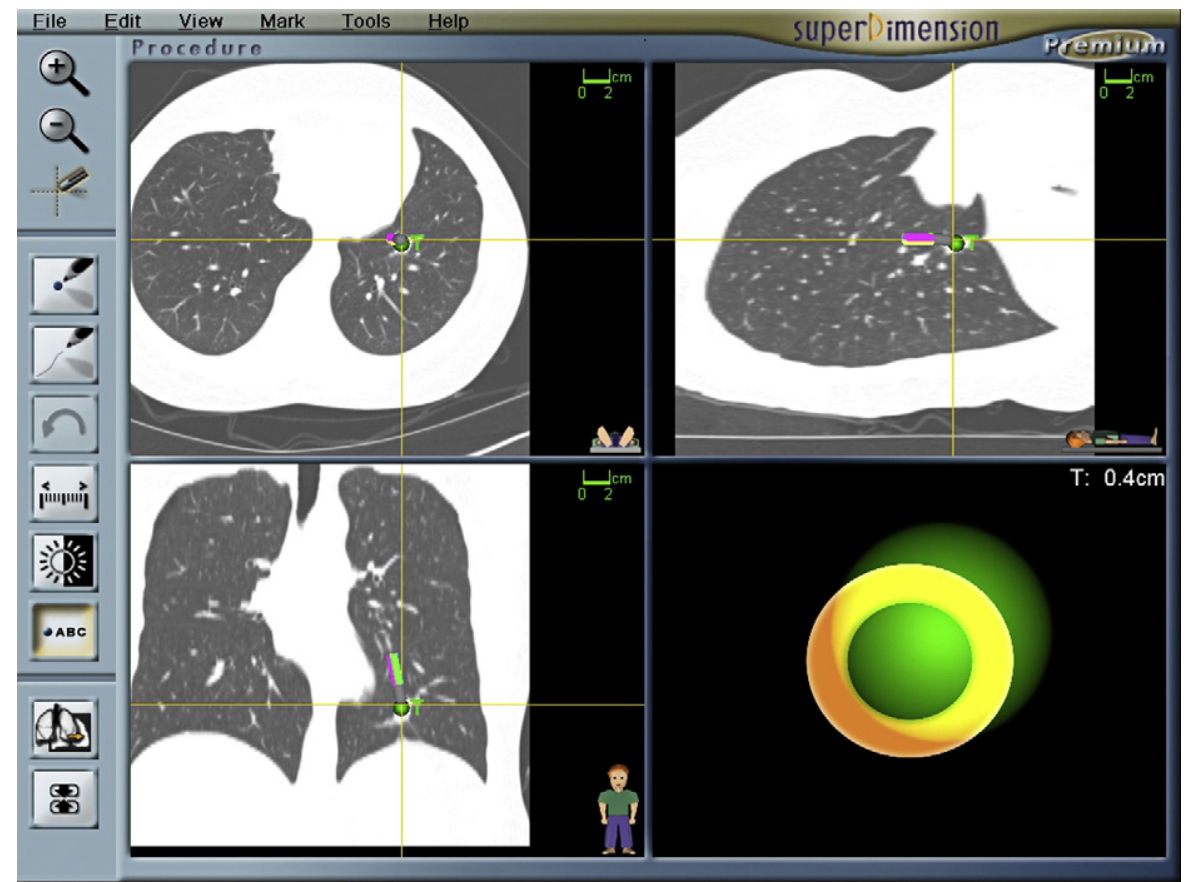

FIGURE 1. Navigation of electromagnetic navigation bronchoscopy (ENB) sensor probe using multiplanar computed tomography $(C T)$ images and "tip-view" orientation. Locator tip was $0.4 \mathrm{~cm}$ from center of targeted tumor location.

only $15 \%$ to $35 \% .^{9,13,14}$ Stereotactic radiosurgery allows for the delivery of high, potentially curative, doses of focused radiation to such localized cancers. Tumor motion in patients with lung cancer is a major obstacle to stereotactic body radiosurgery. Multiple techniques, including breath holding, abdominal compression, 4dimensional excursion prediction, and real-time tumor tracking, are used to guide radiotherapy. ${ }^{15}$ The CyberKnife with Synchrony technology uses real-time respiratory motion tracking with placement of dense metal (gold or platinum) fiducial markers in and near the tumor. ${ }^{16,17}$ Although, in theory, real-time fiducial targeting might be more precise than the nonfiducial-based targeting used by other systems, their accuracy and effectiveness have, to date, not been compared. Although all patients were able to receive complete CyberKnife therapy without the need of any additional fiducial markers, we cannot make any statements regarding treatment efficacy because the median follow-up period was just 9.3 months. Future reports are planned to determine the clinical success and accuracy of this modality.

Typically, 3 noncoplanar fiducial markers are required, each 2-cm apart. ${ }^{6,17}$ Early in the radiosurgery experience, fiducial markers were placed using percutaneous CT guidance. This required a number of passes, resulted in
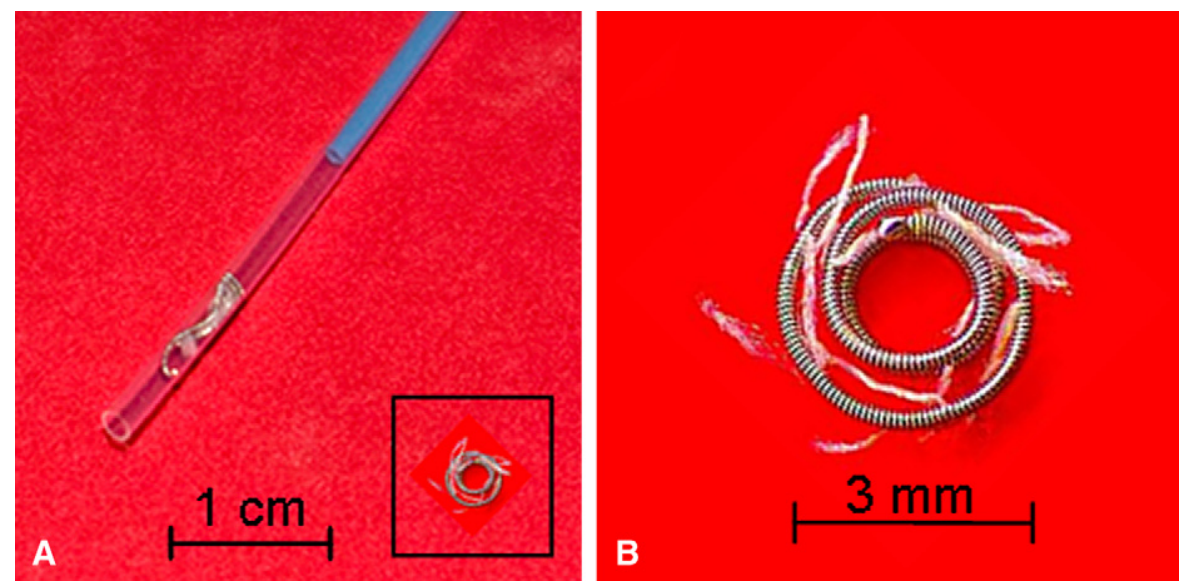

FIGURE 2. A, Flattened platinum coil spring fiducial marker loaded in tip of microbiology specimen brush. Box insert: Deployed coil spring fiducial marker. B, Magnified diamond-shaped deployed coiled spring fiducial marker with thrombogenic filament attachments. 

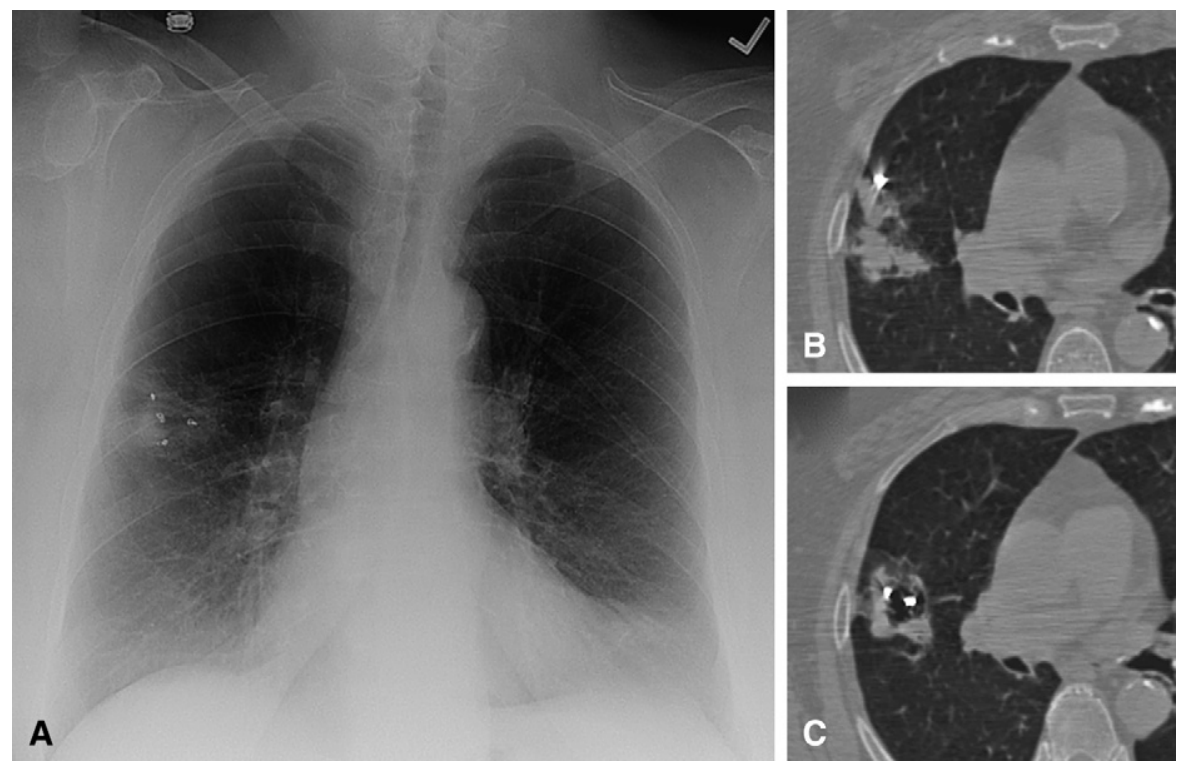

FIGURE 3. Radiopaque fiducial markers identified in and around right lung legion on A, postelectromagnetic navigation bronchoscopy (ENB) chest radiograph, and $\mathrm{B}$ and $\mathrm{C}$, corresponding computed tomography $(C T)$ scan.

a predictable pneumothorax rate of $13 \%$ to $48 \%$, and a frequent need for pigtail chest tube placement. ${ }^{7-9}$ Patients with severe COPD have a very limited ability to tolerate induced pneumothorax and could heal slowly, requiring prolonged hospitalization. Using ENB, the pneumothorax rate in our study was only $5.8 \%$. Most of our patients had COPD, and 7 had 2 tumors. All 3 tumors were pleural-based and 2 had had simultaneous ENB transbronchial biopsy specimens taken. Our pneumothorax rate was within the expected published range of $2 \%$ to $6 \%$ for ENB biopsy of peripheral lung lesions. ${ }^{11,12}$

The deployment of fiducial markers using CT guidance has frequently been suboptimal. For more centrally located tumors, this technique can be hazardous and is generally avoided. Standard flexible fiberoptic bronchoscopy has been used for fiducial placement in central lesions, but it has obvious limitations for accessing peripheral tumors, even with fluoroscopy. ${ }^{9}$ Furthermore, fiducial markers placed with standard bronchoscopic techniques have had a significant premature deployment or "drop" rate $(22 \%)$ owing to the absence of specialized tools and markers. ${ }^{18}$

The commonly used linear gold fiducial markers have a very smooth surface and are frequently dislodged, usually by coughing. Even with ENB techniques in very experienced hands, linear fiducial markers become dislodged $10 \%$ to more than $20 \%$ of the time. ${ }^{10,11,18}$ In our first 4 patients, we lost more than $50 \%$ of the standard linear gold fiducial markers. Therefore, we looked for fiducial markers that would have better seating properties. We have proposed the use of coil spring platinum markers, which are commonly used in endovascular procedures. Platinum is similar in density to the commonly used gold fiducials and can be tracked using the CyberKnife system. These fiducials expand after deployment into a diamondshaped structure and wedge themselves by recoil forces into the surrounding lung tissue. Furthermore, dense polyester fibers attached to the metal coil promote thrombosis and seating into the adjacent tissue. In the subsequent 56 procedures, 217 fiducial markers were placed and only 2 were dislodged $(<1 \%)$. Comparing the dislodgement rate of our coil fiducials with that of linear-type fiducial markers reported by Anantham and colleagues ${ }^{10}(4 / 39,10 \%)$ and Harley and colleagues ${ }^{11}(22 / 161,13.6 \%)$, our coil fiducials were significantly less likely to become dislodged $(P=.0056$ and $P=.0001$, respectively) (Figure 4 ). All patients receiving coil fiducial markers were able to undergo radiosurgery without placement of any additional fiducial markers.

ENB can be performed as an outpatient procedure with the patient under moderate sedation. The procedure was well tolerated, and the procedure time was within the normal limits for interventional bronchoscopic procedures. ${ }^{10,11,19-21}$ General anesthesia has been used at other institutions and carries its own risks. ${ }^{11,12}$ The need for anesthesia staff resources and the added associated costs of general anesthesia favor the use of an interdisciplinary bronchoscopy suite setting.

ENB-guided fiducial marker placement for CyberKnife treatment of peripheral lung tumors is a safe and precise alternative to transthoracic, intravascular, or bronchoscopic modalities currently in use. The steering mechanism of the locator tip and the visual 3-dimensional orientation allow ENB to navigate to a bronchoscopically invisible lesion, where the fiducial markers can be placed with optimal spatial separation. 


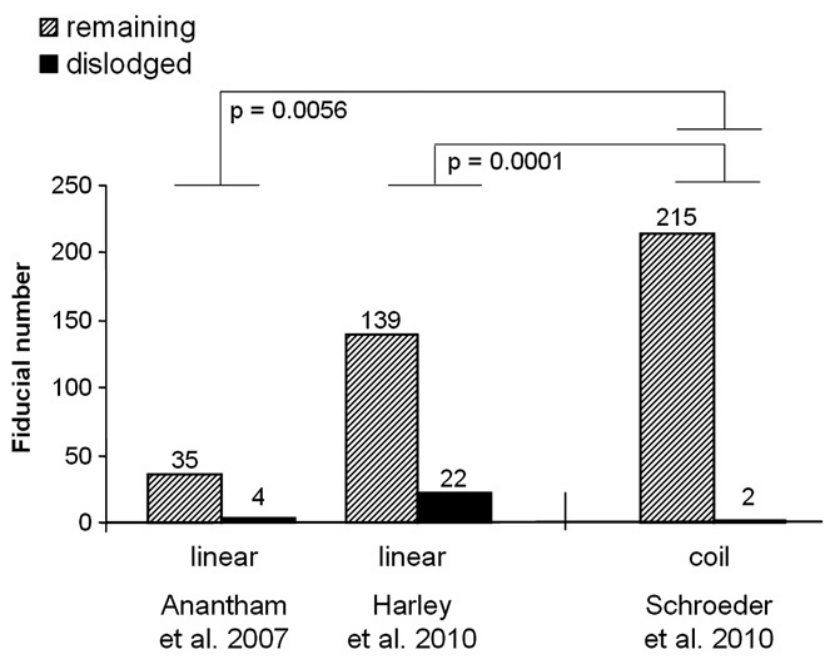

Fiducial Type and Study Source

FIGURE 4. Bar graph depicting remaining and dislodged fiducial markers after electromagnetic navigation bronchoscopy $(E N B)$. Coiled fiducial markers (2 of 217) showed highly significant lower dislodgment rate compared with linear fiducial markers compared with previous studies by Anantham and colleagues ${ }^{10}$ and Harley and colleagues. ${ }^{11} P$ values calculated using Fisher's exact test.

The use of coil spring fiducial markers was $99 \%$ successful for accurate and durable placement. From the published studies, a fiducial loss rate of more than $10 \%$ has been common and results in a greater need of repeat placements and/or a suboptimal CyberKnife tracking accuracy. The deployment of coil spring fiducial markers using a common specimen brush is easy to perform for any trained bronchoscopist. The coil spring fiducial markers are more expensive (about $\$ 104$ vs \$65) than the classic linear type. The lower dislodgement rate should negate the need for repeat fiducial placement ${ }^{10}$ and its attendant costs in materials, staff, and periprocedural risk.

\section{References}

1. Brown WT, Wu X, Fayad F, Fowler JF, Amendola BE, García S, et al. CyberKnife radiosurgery for stage I lung cancer: Results at 36 months. Clin Lung Cancer. 2007;8:488-92.

2. Brown WT, Wu X, Fowler JF, García S, Fayad F, Amendola BE, et al. Lung metastases treated by CyberKnife image-guided robotic stereotactic radiosurgery at 41 months. South Med J. 2008;101:376-82.

3. Christie NA, Pennathur A, Burton SA, Luketich JD. Stereotactic radiosurgery for early stage non-small cell lung cancer: Rationale, patient selection, results, and complications. Semin Thorac Cardiovasc Surg. 2008;20:290-7.

4. Collins BT, Vahdat S, Erickson K, Collins SP, Suy S, Yu X, et al. Radical CyberKnife radiosurgery with tumor tracking: an effective treatment for inoperable small peripheral stage I non-small cell lung cancer. J Hematol Oncol. 2009;2:1.

5. Pennathur A, Luketich JD, Heron DE, Schuchert MJ, Burton S, Abbas G, et al. Stereotactic radiosurgery for the treatment of lung neoplasm: Experience in 100 consecutive patients. Ann Thorac Surg. 2009;88:1594-600.

6. Whyte RI, Crownover R, Murphy MJ, Martin DP, Rice TW, DeCamp MM Jr, et al. Stereotactic radiosurgery for lung tumors: Preliminary report of a phase I trial. Ann Thorac Surg. 2003;75:1097-101.

7. Geraghty PR, Kee ST, McFarlane G, Razavi MK, Sze DY, Dake MD. CT-guided transthoracic needle aspiration biopsy of pulmonary nodules: Needle size and pneumothorax rate. Radiology. 2003;229:475-81.
8. Yeow KM, Su IH, Pan KT, Tsay PK, Lui KW, Cheung YC, et al. Risk factors of pneumothorax and bleeding: Multivariate analysis of $660 \mathrm{CT}$-guided coaxial cutting needle lung biopsies. Chest. 2004;126:748-54.

9. Pennathur A, Luketich JD, Heron DE, Abbas G, Burton S, Chen M, et al. Stereotactic radiosurgery for the treatment of stage I non-small cell lung cancer in highrisk patients. J Thorac Cardiovasc Surg. 2009;137:597-604.

10. Anantham D, Feller-Kopman D, Shanmugham LN, Berman SM, DeCamp MM, Gangadharan SP, et al. Electromagnetic navigation bronchoscopy-guided fiducial placement for robotic stereotactic radiosurgery of lung tumors: A feasibility study. Chest. 2007;132:930-5.

11. Harley DP, Krimsky WS, Sarkar S, Highfield D, Aygun C, Gurses B. Fiducial marker placement using endobronchial ultrasound and navigational bronchoscopy for stereotactic radiosurgery: An alternative strategy. Ann Thorac Surg. 2010;89:368-74

12. Eberhardt R, Anantham D, Ernst A, Feller-Kopman D, Herth F. Multimodality bronchoscopic diagnosis of peripheral lung lesions: A randomized controlled trial. Am J Respir Crit Care Med. 2007;176:36-41.

13. Nuyttens JJ, Prévost JB, Praag J, Hoogeman M, Van Klaveren RJ, Levendag PC, et al. Lung tumor tracking during stereotactic radiotherapy treatment with the CyberKnife: Marker placement and early results. Acta Oncol. 2006;45:961-5.

14. Qiao X, Tullgren O, Lax I, Sirzén F, Lewensohn R. The role of radiotherapy in treatment of stage I non-small cell lung cancer. Lung Cancer. 2003;41:1-11.

15. Timmerman R, Paulus R, Galvin J, Michalski J, Straube W, Bradley J, et al. Stereotactic body radiation therapy for inoperable early stage lung cancer. JAMA. 2010;303:1070-6.

16. Schweikard A, Shiomi H, Adler J. Respiration tracking in radiosurgery. Med Phys. 2004;31:2738-41.

17. Murphy MJ. Fiducial-based targeting accuracy for external-beam radiotherapy. Med Phys. 2002;29:334-44

18. Reichner CA, Collins BT, Gagnon GJ, Malik S, Jamis-Dow C, Anderson ED. The placement of gold fiducials for CyberKnife stereotactic radiosurgery using a modified transbronchial needle aspiration technique. J Bronchol. 2005;12:193-5.

19. Eberhardt R, Anantham D, Herth F, Feller-Kopman D, Ernst A. Electromagnetic navigation diagnostic bronchoscopy in peripheral lung lesions. Chest. 2007;131: 1800-5.

20. Schwarz Y, Greif J, Becker HD, Ernst A, Mehta A. Real-time electromagnetic navigation bronchoscopy to peripheral lung lesions using overlaid CT images: The first human study. Chest. 2006;129:988-94.

21. Gildea TR, Mazzone PJ, Karnak D, Meziane M, Mehta AC. Electromagnetic navigation diagnostic bronchoscopy: A prospective study. Am J Respir Crit Care Med. 2006;174:982-9.

\section{Discussion}

Dr Bryan Fitch Meyers (St. Louis, Mo). I see that there are actually several alternatives, and you mentioned some. You could use percutaneous placement of these fiducials with a greater risk of pneumothorax, but presumably that could be done with just a local anesthetic. Your method has a pneumothorax risk as well, albeit lower, but it would require more sedation or anesthetics, and people would have to weigh the pros and cons of those different approaches. What was the sedation or anesthetic that you gave in all or most of these patients, and could you just talk a little bit about the setting of the procedure and the duration of the procedure?

Dr Schroeder. The maximum duration was up to 1 hour. We got better with the learning curve. If it is just a straight fiducial placement, we are down to a 20-minute procedure. It all depends on whether you actually have an easy way to reach the tumor. The patients are in moderate sedation in an out-patient setting. We actually tap into the system of our pulmonology colleagues, and fentanyl and midazolam is normally used.

I honestly think the lower rate of pneumothorax is worthwhile to undergo a moderate sedation protocol. You see in some studies every other patient has a pneumothorax with the treatment of a pigtail catheter and being in the hospital. I think that is an important comorbidity. 
Dr Robert J. Cusimano (Toronto, Ontario, Canada). Just a small question. I am a cardiac surgeon so I do not know about these things. The coil markers, are they ferromagnetic? Can you perform magnetic resonance imaging afterward?

Dr Schroeder. Yes, you can perform magnetic resonance imaging. They stay in. They are platinum.

Dr Arjun Pennathur (Pittsburgh, Pa). Great presentation. How many patients actually underwent biopsy that was proven? It looked like on your last slide you said about $45 \%$. So were other $55 \%$ still going to undergo transthoracic computed tomography (CT)-guided biopsy? How did you perform biopsies on the rest of the patients?
Dr Schroeder. We are a secondary center, so we often have people referred from the outside who had undergone bronchoscopy or CT-guided biopsy beforehand.

Dr Pennarthur. How was your success rate for a peripheral lesion with a navigational bronchoscopy to get a tissue diagnosis?

Dr Schroeder. Our biopsy rate is about in the 50\% range. I know that in other hands with navigational bronchoscopy alone it is in the $70 \%$ range. So, our rate was about $50 \%$, unfortunately.

Dr Pennarthur. So, in those patients you would still perform CT-guided biopsy?

Dr Schroeder. Or a repeat bronchoscopy is another possibility. 